\title{
Unplanned Readmissions after Spine Surgery: A Single-Center Prospective Analysis of a 90-Day Model in 2,860 Cases
}

\author{
Mahender Avinash, Karukayil Ramakrishnan Renjith, Ajoy Prasad Shetty, \\ Vyom Sharma, Rishi Mugesh Kanna, Shanmuganathan Rajasekaran \\ Department of Orthopedics, Ganga Medical Centre « Hospital Pvt. Ltd., Coimbatore, India
}

\begin{abstract}
Study Design: Prospective study.
Purpose: During the last decades, an emergence of unplanned readmissions has been shown to be a useful tool to gage the healthcare quality and hospital performance. Previous studies were limited by their retrospective designs based on database information and short-term 30-day follow-up intervals. We analyzed the incidence and causes for unplanned readmissions following spine surgery at a 90-day interval and the difference at 30-, 31-60-, and 61-90-day intervals after discharge. Additionally, we assessed total beddays lost and the economic impact of readmissions and probable risk factors.

Overview of Literature: Recent reports on readmission rates suggested the contribution of this parameter for the assessment of healthcare quality.

Methods: A prospective analysis of 2,860 admissions was performed over 1 year in a tertiary care orthopedic hospital. All unscheduled readmissions following spine surgery within 90 days of discharge were included, irrespective of type or location of surgery. Polytrauma, primary osseous infections, and planned readmissions were excluded.

Results: Our readmission rate was 3.32\% (95/2,860). Leading readmission causes were surgical site infections (SSIs) accounting for 44.21\% ( $n=42$; superficial, 23; deep, 11; organ and space, 8), followed by aseptic pain $31.58 \%$ ( $n=30)$ and medical causes 13.68\% ( $n=13$ ). Though $86.95 \%$ of superficial SSIs occurred within 30 days, $21.1 \%$ of deep SSIs occurred beyond 30 days. During the $30-90$-day interval, $33.68 \%$ of readmissions occurred. The financial burden amounted to 41,93,660 Indian Rupees, and the mean bed-days lost was 7.33 per readmission. Hospital stay $\geq 10$ days, health insurance, and comorbid illnesses (diabetes, hypertension, and liver disease) were associated with readmissions $(p<0.05)$.

Conclusions: Our study showed that SSIs and aseptic pain were the leading causes of readmissions at 90 days after spine surgery. Limiting the analysis to 30-day readmissions as in previous studies would lead to failure in the identification of more severe complications like deep SSIs. Continued vigilance, particularly for patients with predisposing factors, could help alleviate the financial burden.
\end{abstract}

Keywords: Readmissions; Quality of health care; Surgical-site infections; Spine

Received Mar 25, 2019; Accepted Apr 22, 2019

Corresponding author: Ajoy Prasad Shetty

Department of Spine Surgery, Ganga Medical Centre \& Hospital Pvt. Ltd., Coimbatore, Tamil Nadu, India

Tel: +91-9344833797, Fax: +91-422241444, E-mail: ajoyshetty@gmail.com 


\section{Introduction}

Unplanned readmission rates following surgery gained considerable interest over the last decade because these can be a useful marker of healthcare quality, hospital performance, and cost-effectiveness. Studies have shown that as many as one in four patients returns to the hospital within 30 days of discharge, and the estimated costs in Medicare spending due to readmissions were reported to be as high as $\$ 17.6$ billion [1-3]. With the introduction of the Hospital Readmissions Reduction Program, the centers for Medicare and Medicaid services can even penalize hospitals for higher readmission rates; therefore, every effort has been directed toward establishing a system to reduce the risk of readmission, which would help in curtailing the costs for the healthcare system [4].

The 30-day readmission rates in orthopedics have been reported to be between $2 \%-14 \%$. In spine surgery, readmission rates range from $2.5 \%$ to as high as $9.42 \%$ given the variance of procedures studied [5-8]. However, most of these spine surgery studies were retrospective, limited to readmissions within a 30-day interval postoperatively, and the vast majority were based on elective spine surgery cases only using a specific procedure or location [7,9-14]. The existing literature reporting on 90-day readmission rates was predominantly based on a national administrative database, which is frequently error-prone due to under-reporting and lack of clinical details [15]. The available reports on economic impact analysis are most often focused on Medicare-based spending and relate specifically to total joint arthroplasty procedures, without giving a perspective on out-of-pocket expenditures [9]. In developing countries, where healthcare insurance coverage is negligible, this out-of-pocket expenditure can prove to be a substantial financial burden to the patient $[16,17]$.

To the best of our knowledge, no previous studies analyzed hospital readmissions or their economic impact in developing countries. Moreover, a 30-day interval is considered an overly short period to properly determine the consequences of a major surgical procedure $[18,19]$. With this as background, we performed a prospective analysis of unplanned readmissions within 90 days following spine surgery, regardless of the type or location of the procedure, in an orthopedic specialty unit. We sought to evaluate (1) the incidence and causes for unplanned readmissions at a 90-day interval and its difference at 30-, 31-60-, and 61-90-day intervals; (2) total bed-days lost (TBL) and economic impact of readmissions; and (3) probable risk factors.

\section{Materials and Methods}

\section{Patient selection}

We performed a prospective analysis of 2,860 consecutive patients admitted to the spine unit of Ganga Medical Centre \& Hospital Pvt. Ltd., Coimbatore, India, between 1 January 2015 to 1 January 2016. The study was approved by the Institutional Review Board of Ganga Medical Centre and Hospitals Pvt. Ltd., Coimbatore (IRB approval no., 2015/10/02, dated 09/11/2015), and informed consent was obtained from all readmitted patients before retrieving any relevant information from their medical records. All patients receiving unscheduled readmissions following spine surgery within 90 days of discharge, irrespective of the type or location of surgery, were included. We excluded all patients readmitted for planned staged procedures, patients with polytrauma, and patients admitted with primary osseous infections (without previous surgical procedure). The mean age of the population was 46.02 \pm 5.3 years (range, 2-94 years), which included 1,758 males (61.4\%) and 1,102 females (38.6\%).

\section{Data collection}

The readmissions were identified daily from the hospital admissions register by an orthopedic surgeon (first author). The timing of the readmission was calculated based on the time from discharge after the index hospitalization. Readmissions were classified as having surgical and nonsurgical causes. Surgical causes included surgical site infections (SSI), aseptic pain (back pain or radiculopathy caused by factors unrelated to infections such as recurrent disk prolapse), implant-related complications, and readmissions due to recurrence or worsening of the neurodeficit. Nonsurgical causes could be medical complications like urinary tract infection, uncontrolled diabetes, or deep vein thrombosis (DVT). TBL was calculated as the total sum of the length of the hospital stay for each readmission. The economic impact was based on the direct expenses in Indian Rupee (INR) incurred by the patient during the period of readmission, which was inclusive of room charges, surgeon fees, nursing charges, and surgical procedure costs. However, the indirect expenses arising 
out of the loss of productivity and unemployment were not included. Relevant data regarding patient demographics and comorbidities, insurance coverage, and duration of hospital stay during the index procedure were also obtained using the Health Object extraction software (Idea Objects, Chennai, India).

\section{Outcome measures}

Our primary outcome measures were readmission rates at the 90-day interval and the differences in incidence and causes for readmission at 30-, 31-60-, and 61-90-day intervals postoperatively. The secondary outcomes included TBL and economic impact as well as the probable risk factors leading to readmission.

\section{Statistical analysis}

Categorical variables are expressed as percentages or total numbers. A statistical analysis of categorical variables was done using the chi-square or $t$-test. A $p$-value less than 0.05 (confidence interval, 95\%) was considered significant.

\section{Results}

\section{Primary outcome}

1) Rates and causes of readmissions within 90-day postoperatively

Of the 2,860 admissions, we noticed an unplanned readmission rate of $3.32 \%$ at the 90 -day interval. During this period, there were 95 readmissions in 85 patients, and five patients were readmitted twice within the 90-day interval. The causes of readmission and the corresponding percentages are summarized in Table 1.

Surgical causes were the leading causes of readmission, with SSIs (42.4 \%) being the most common followed by aseptic pain (31.58\%). In contrast, medical causes constituted $13.68 \%$. Among the superficial SSIs $(n=23)$, wound debridement and secondary suturing were required in 21 cases while two patients improved by conservative management with intravenous antibiotics. All deep SSIs required wound exploration and debridement; one patient required further surgical instrumentation owing to a loosened L5 screw, and one patient required vacuum-assisted wound closure to achieve complete wound closure. All organ and space infections were managed with debride-
Table 1. Causes and proportions of readmissions at 90-day interval $(\mathrm{N}=2,860)$

\begin{tabular}{lcc}
\hline Variable & \multicolumn{1}{c}{ Causes } & $\begin{array}{c}\text { No. (\%) of } \\
\text { readmissions }\end{array}$ \\
\hline Total & Surgical site infection & $95(3.32)$ \\
\hline Surgical & Superficial & $42(44.21)$ \\
& Deep & 23 \\
& Organ space & 11 \\
& Aseptic pain & 8 \\
& Recurrent disc prolapse & $30(31.58)$ \\
& Implant related & 10 \\
& Recurrence/worsening of & $4(4.21)$ \\
& neurodeficit & $3(3.16)$ \\
& Miscellaneous & $3(3.16)$ \\
\hline Non-surgical & Medical & $13(13.68)$ \\
\hline & &
\end{tabular}

ment. Of these, two patients received additional antibiotic bead application, and one patient required a surgical revision and instrumentation.

There were 30 readmissions for aseptic pain of which 10 were due to recurrent disc prolapse. Seven patients primarily presented with a L4-5 disc disease, two presented with L5-S1 involvement, and one presented with L3-L4, L4-L5, and L5-S1 involvement. Five of these readmissions were managed with nerve root blocks, four required revision decompression and discectomy, while one required a transforaminal lumbar interbody fusion. The remaining 20 readmissions with aseptic pain, their timing, and management were summarized in Tables 2-4.

Four patients were readmitted for implant-related causes, which included malpositioned pedicle screws $(n=2)$, posterior migration of interbody cages $(n=1)$, and pedicle screw back-out $(n=1)$. In all these cases, management of the implant-related causes required a procedure in the operating room. The miscellaneous causes included two readmissions for catheter blockage and one for pressure sore care. Among the nonsurgical causes $(n=13)$, there were five events of urinary tract infection, three events of respiratory tract infection, three uncontrolled diabetes, and two events of DVT.

2) Difference between the incidence and causes of readmission at 30-, 60-, and 90-day intervals Most readmissions occurred within 30 days of discharge $(66.32 \%, n=63)$. The readmission rates at $31-60$ - and 
Table 2. Cause for aseptic pain and its management at 30-day interval (excluding recurrent discs)

\begin{tabular}{lllll} 
No. & \multicolumn{1}{c}{ Primary procedure } & Readmission pain type & \multicolumn{1}{c}{ Cause of pain } & Treatment \\
\hline 1 & $\begin{array}{c}\text { Failed L5-S1 microdiscectomy, reexploration } \\
\text { loose fragment removal and selective nerve } \\
\text { root block }\end{array}$ & Back and radiculopathy & Unknown & Symptomatic \\
\hline 2 & L3-4 microdiscectomy & Radiculopathy & Unknown & Symptomatic \\
\hline 3 & $\begin{array}{l}\text { Following L4-S1 posterior decompression and } \\
\text { fusion (epidural fibrosis around L5 nerve root) }\end{array}$ & Back & MRI: seroma, dural tear & Exploration and sealing the \\
tear
\end{tabular}

$\mathrm{MRI}$, magnetic resonance imaging.

Table 3. Cause for aseptic pain and its management at 31-60-day interval (excluding recurrent discs)

\begin{tabular}{|c|c|c|c|c|}
\hline No. & Primary procedure & Readmission pain type & Cause of readmission pain & Treatment \\
\hline 1 & L5-S1 TLIF & $\begin{array}{l}\text { Back+radiculopathy following } \\
\text { sneezing }\end{array}$ & $\begin{array}{l}\text { Residual L5-S1 anterolisthesis with } \\
\text { left L5 pedicular lysis and ipsilateral } \\
\text { exit foramen narrowing and seroma }\end{array}$ & $\begin{array}{l}\text { Reexploration and exten- } \\
\text { sion of instrumentation to } \\
\text { L4 }\end{array}$ \\
\hline 2 & TLIF for $L 4-5$ recurrent disc & Radiculopathy & Hematoma around nerve root & Nerve root block \\
\hline 3 & L5-S1 microdiscectomy & Radiculopathy & Hematoma around nerve root & Nerve root block \\
\hline 4 & L5-S1 TLIF & Radiculopathy & Suspected arachnoiditis & Nerve root block \\
\hline 5 & C4 partial corpectomy and C4-5 ACDF & Hand pain/numbness & Carpal tunnel syndrome & Carpal tunnel release \\
\hline 6 & L3-4 decompression & Radiculopathy & Unknown & Nerve root block \\
\hline 7 & L5-S1 TLIF & Radiculopathy & L5-S1 nerve root adhesion & Exploration and neurolysis \\
\hline
\end{tabular}

TLIF, transforaminal lumbar interbody fusion; ACDF, anterior cervical discectomy and fusion.

Table 4. Cause for aseptic pain and its management at 61-90-day interval (excluding recurrent discs)

\begin{tabular}{lllll} 
No. & \multicolumn{1}{c}{ Primary procedure } & $\begin{array}{c}\text { Readmission } \\
\text { pain type }\end{array}$ & Cause of readmission pain & Treatment \\
1 & L5-S1 decompression and microdiscectomy & Back & Mechanical & Symptomatic \\
\hline 2 & D11-L2 instrumentation for D12 carrot stick fracture & Back & Fracture through D11 pedicle screw & Reinstrumentation \\
\hline $3 \& 4$ & L4-L5 transforaminal lumbar interbody fusion & Back & Mechanical & Symptomatic \\
\hline
\end{tabular}

61-90-days were $23.16 \%(n=22)$ and $10.53 \%(n=10)$, respectively. Among the surgical factors, the leading cause at the 30-day interval was SSI (55.5\%), while aseptic pain was the major reason at 31-60-days (40.9\%) and 61-90- days intervals (60\%). A summary of the difference in incidence and major causes for readmissions at varying time intervals are detailed in Table 5. 
Table 5. Difference in incidence and major causes for readmissions at 30-, 31-60-, and 61-90-day intervals

\begin{tabular}{|c|c|c|c|}
\hline \multirow{2}{*}{$\begin{array}{l}\text { Causes for } \\
\text { readmissions }\end{array}$} & \multicolumn{3}{|c|}{ Readmission interval (day) } \\
\hline & $<30$ & $31-60$ & $61-90$ \\
\hline Total no. (\%) & $63(66.32)$ & $22(23.16)$ & 10 (10.53) \\
\hline Surgical site infections & $35(55.5)$ & $7(31.8)$ & - \\
\hline Superficial & 20 & 3 & - \\
\hline Deep & 9 & 2 & - \\
\hline Organ space & 6 & 2 & - \\
\hline Aseptic pain & $15(23.8)$ & $9(40.9)$ & $6(60)$ \\
\hline Recurrent disc prolapse & 6 & 2 & 2 \\
\hline Medical causes & $8(12.7)$ & $3(13.6)$ & $2(20)$ \\
\hline Urinary tract infection & 2 & 1 & 2 \\
\hline Deep vein thrombosis & - & 1 & - \\
\hline
\end{tabular}

Values are presented as number (\%) or number.

Table 6. Analysis of probable risk factors

\begin{tabular}{lc} 
Risk factor & $p$-value \\
Age $\geq 70$ yr & 0.93 \\
\hline Length of stay $\geq 10$ days & $<0.001^{*}$ \\
\hline Gender & 0.24 \\
\hline Health insurance & $0.007^{*}$ \\
\hline Comorbid illnesses & \\
\hline Diabetes & $0.035^{*}$ \\
\hline Hypertension & $0.001^{*}$ \\
\hline Hypothyroidism & 0.943 \\
\hline Ischemic heart disease & 0.507 \\
\hline Liver disease & $0.001^{*}$ \\
\hline
\end{tabular}

$p<0.05$; statistically significant.

\section{Secondary outcome}

1) TBL and economic impact

TBL secondary to readmissions was 688 days overall, and the mean bed-days lost was $7.33 \pm 1.5$ days per patient (range, 1-28 days). Direct costs arising out of 90-day readmissions was INR 41,93,660 with a mean expenditure of INR 44,143.79 per readmission (range, INR 2,625223,955).

\section{2) Analysis of probable risk factors}

Our study documented that prolonged hospital stay $\geq 10$ days during the index procedure $(p=0.001)$, health insurance coverage $(p=0.007)$, and comorbid illnesses such as diabetes $(p=0.035)$, hypertension $(p=0.001)$, and liver dis- ease $(p=0.001)$ were significant risk factors for readmission. The detailed results of the statistical analysis for each risk factor are shown in Table 6.

\section{Discussion}

Recently, a number of studies investigated various aspects of unplanned readmissions after surgery to prevent "higher than expected" readmission rates. We conducted a prospective analysis of the readmission rates following spine surgery and its causes within the 90-day postoperative interval at a single-center. Additionally, our study attempted to identify the difference in readmission rates and its causes at 30-, 31-60-, and 61-90-day intervals following discharge along with its financial impact and probable predisposing factors.

At our institution, we had a 90 -day readmission rate of $3.32 \%$ in 2,860 patients, which included patients of all ages receiving either elective and emergency spine procedures. The lowest readmission rates in spine surgery were reported by Lovecchio et al. [7] at 2.6\%, anterior cervical discectomy, and fusion in 2,320 patients. In contrast, the highest reported rate was $9.42 \%$ following elective spine surgery in 1,400 patients, as reported by Adogwa et al. [8]. Both of the above-mentioned studies were retrospective studies focusing on patients readmitted at the 30-day intervals postoperatively, whereas ours was a prospective study on a higher sample size at the 3-month interval following discharge.

In a multi-centric retrospective analysis of readmissions in lumbar spine surgery, Pugely et al. [12] found that the main reasons for readmissions were wound-related (38.6\%) and pain-related $(22.4 \%)$ causes followed by thromboembolism (9.4\%) and systemic infections (8.0\%). In 2012, McCormack et al. [20] conducted a single-center study on 3,673 cases and reported that infections (32\%) were the leading cause of readmissions while medical problems (excluding DVT) accounted for $22 \%$ of readmissions. Another single-center retrospective study by Adogwa et al. [8] that followed up 1,400 patients noted that SSIs (34.8\%), pain (19.7\%), and fever (16.7\%) were the three main causes for readmission following elective spine surgery. Analogous to the above-mentioned studies, our current study also showed that SSI $(n=42,44.21 \%)$ was the most common cause for readmission followed by persistent pain $(n=30,31.58 \%)$ and medical causes $(n=13$, $13.68 \%$ ). The low rate of medical readmissions could be 
attributed to the fact that our center is a specialized orthopedic institute, unlike the other multispecialty centers in which the study population might include a higher number of patients with comorbid illnesses as well.

There are a paucity of data regarding the differences in incident rates and causes for readmissions at varying intervals following surgery. Within 30 days of discharge, the total percentage of readmission was $66.32 \%$. This implies that a significant $33.68 \%$ occurred beyond this interval (23.16\%, $\mathrm{n}=22$ in 31-60-day interval; $10.53 \%, \mathrm{n}=10$ in the 61-90-day interval), which further emphasizes the importance of a longer follow-up model. Most of the Medicare bundled payment plans define the immediate postoperative period as 3 months. Moreover, in a tertiary care center like ours, follow-up appointments are given 4 to 6 weeks in advance from the date of discharge; therefore, a 90 day model would be more ideal to assess hospital performance. While SSIs remained the most common cause for readmission at the 30-day interval, aseptic pain was the leading cause in 31-60- and 61-90-day intervals (40.9\% and $60 \%$, respectively). We consider that this finding is clinically relevant as it could be useful for performing targeted interventions at the appropriate timing, thereby decreasing the readmission rates to a minimum.

With 688 bed-days being lost to readmissions and a mean of $7.33 \pm 1.5$ days per readmission, a considerable number of beds essential for other elective and emergency admissions were occupied during the hospital stay of our patients. In 2004, the estimated expense due to unplanned readmissions was approximately $\$ 17.6$ billion in Medicare spending $[1,3]$. The financial impact of readmissions was INR 41,93,660 during the study period with a mean of INR 44,143.79 (range, INR 2,625 to 2,23,955) being spent per patient. Most inpatients were from low socio-economic strata as evidenced by a mean per capita income of INR 13,400 in our study group. Besides, the Central Statistics Office on the Advance Estimate of National Income, 2012-2013 showed that India's per capita monthly income was only INR 5,729, re-iterating the substantial economic burden posed by readmissions of patients $[16,17]$. We also noted that only $14.74 \%$ of the readmitted patients in our study population had health insurance coverage and none of them covered the expenses met during the readmission. In a population where a vast majority of individuals pay for healthcare from their own pockets, unplanned readmissions prove to be a costly financial burden, especially when the majority of patients belong to a lower income group $[16,17]$. The only authors who analyzed the economic impact of readmissions were Clement et al. [9]. They found that if Medicare stops reimbursing readmissions, the institution under review might sustain an average loss of $\$ 11,494$ [9]. However, their study group comprised only of patients with total hip arthroplasties.

In a meta-analysis, Bernatz et al. [21] found that age, length of stay, discharge to a skilled nursing facility, increased body mass index, the American Society of Anesthesiologists (ASA) score $\geq 3$, and Medicare and Medicaid insurance demonstrated a statistically positive correlation with increased 30 -day readmissions in $\geq 75 \%$ of studies. Pugely et al. [12] observed that the predictors of readmission after lumbar spine surgery included advanced patient age $\geq 80$ years, African American race, recent weight loss, chronic obstructive pulmonary disorder, history of cancer, creatinine levels $\geq 1.2$, high ASA class, operative time $>4$ hours, and prolonged hospital stay for more than 4 days. In 2018, Elsamadicy et al. [22] reported that insurance status, having chronic obstructive pulmonary disorder, depression, hypertension, diabetes, obesity, or deficiency anemia were independently associated with 90-day readmissions following elective spine surgery. Readmissions in our series showed a statistically significant association with prolonged hospital stay ( $\geq 10$ days) $(p<0.001)$, health insurance ( $p=0.007)$, comorbid illnesses (diabetes [ $p=0.035]$, hypertension $[p=0.001]$, and liver disease $[p<0.001])$ in accordance with the above-mentioned literature (Table 5).

This study has some limitations such as the fact that multivariate logistic regression analysis was not performed in our study to determine independent risk factors for readmissions at specific time intervals. The emergency department visits of patients were not tracked, and underreporting could be possible as patients with nonsurgical complications might seek medical attention at some other institution.

We believe that a 3-month interval, rather than a 30-day interval, is ideally suited for readmission analysis, especially for cases of spine surgery, in which the advantages of covering the immediate postoperative period of Medicare bundled plans as well as major adverse events that could reflect the quality of healthcare and hospital performance. In this prospective study, we provide a unique perspective on unplanned readmissions occurring within 90 days postoperatively in patients of all ages receiving spine surgery by analyzing the clinical setting of a tertiary 
care spine unit in a developing nation.

\section{Conclusions}

Our study showed that the leading causes of readmissions following spine surgery were surgical causes, including SSIs and aseptic causes of postoperative pain. Our 90-day model identified an additional $32(33.68 \%)$ readmissions, which included $21.1 \%$ for deep SSIs. We confirmed the importance of a 90-day study as compared to the routine 30-day analysis. The analysis of varying causes at different time intervals can allow targeted interventions at the appropriate timing which could lead to an improved quality of the healthcare delivery and reduce the financial burden on the patient.

\section{Conflict of Interest}

No potential conflict of interest relevant to this article was reported.

\section{Author Contributions}

Conceptualization: MA, KRR, APS, RMK, SR; data curation: MA, VS; formal analysis: MA, KRR, APS, VS, RMK, SR; methodology: MA, KRR, APS; software: MA; writingoriginal draft: MA, KRR, APS; writing-review \& editing: VS, RMK, RS; final approval of the version: MA, KRR, APS, VS, RMK, RS; supervision: KRR, APS, VS; validation: KRR, APS, VS; and project administration: RMK, SR.

\section{References}

1. Jencks SF, Williams MV, Coleman EA. Rehospitalizations among patients in the Medicare fee-for-service program. N Engl J Med 2009;360:1418-28.

2. Stefan MS, Pekow PS, Nsa W, et al. Hospital performance measures and 30-day readmission rates. J Gen Intern Med 2013;28:377-85.

3. Medicare Payment Advisory Commission. Report to the congress: promoting greater efficiency in Medicare. Washington (DC): MedPAC; 2007.

4. Boozary AS, Manchin J 3rd, Wicker RF. The Medicare Hospital Readmissions Reduction Program: time for reform. JAMA 2015;314:347-8.

5. Issa $\mathrm{K}$, Cherian JJ, Kapadia BH, et al. Readmission rates for cruciate-retaining total knee arthroplasty. J Knee Surg 2015;28:239-42.

6. Schairer WW, Carrer A, Deviren V, et al. Hospital readmission after spine fusion for adult spinal deformity. Spine (Phila Pa 1976) 2013;38:1681-9.

7. Lovecchio F, Hsu WK, Smith TR, Cybulski G, Kim B, Kim JY. Predictors of thirty-day readmission after anterior cervical fusion. Spine (Phila Pa 1976) 2014;39:127-33.

8. Adogwa O, Elsamadicy AA, Han JL, Karikari IO, Cheng J, Bagley CA. 30-Day readmission after spine surgery: an analysis of 1400 consecutive spine surgery patients. Spine (Phila Pa 1976) 2017;42:520-4.

9. Clement RC, Derman PB, Graham DS, et al. Risk factors, causes, and the economic implications of unplanned readmissions following total hip arthroplasty. J Arthroplasty 2013;28(8 Suppl):7-10.

10. Martin CT, Pugely AJ, Gao Y, Weinstein SL. Causes and risk factors for 30-day unplanned readmissions after pediatric spinal deformity surgery. Spine (Phila Pa 1976) 2015;40:238-46.

11. Amin BY, Tu TH, Schairer WW, et al. Pitfalls of calculating hospital readmission rates based on nonvalidated administrative data sets: presented at the 2012 Joint Spine Section Meeting: clinical article. J Neurosurg Spine 2013;18:134-8.

12. Pugely AJ, Martin CT, Gao Y, Mendoza-Lattes S. Causes and risk factors for 30-day unplanned readmissions after lumbar spine surgery. Spine (Phila Pa 1976) 2014;39:761-8.

13. Anand N, Sardar ZM, Simmonds A, Khandehroo B, Kahwaty S, Baron EM. Thirty-day reoperation and readmission rates after correction of adult spinal deformity via circumferential minimally invasive surgery-analysis of a 7-year experience. Spine Deform 2016;4:78-83.

14. Nacke E, Ramos N, Stein S, Hutzler L, Bosco JA 3rd. When do readmissions for infection occur after spine and total joint procedures? Clin Orthop Relat Res 2013;471:569-73.

15. Wang MC, Kreuter W, Wolfla CE, Maiman DJ, Deyo RA. Trends and variations in cervical spine surgery in the United States: Medicare beneficiaries, 1992 to 2005. Spine (Phila Pa 1976) 2009;34:955-61.

16. La Forgia G, Nagpal S. Government-sponsored health insurance in India: are you covered? Washington (DC): World Bank Publications; 2012. 
17. Insurance Regulatory and Development Authority India. Annual report 2013-14 [Internet]. Hyderabad: Insurance Regulatory and Development Authority India; 2014 [cited 2017 May 1]. Available from: https://www.irdai.gov.in/ADMINCMS/cms/frmGeneral_Layout.aspx?page=PageNo2410\&flag=1.

18. Bohl DD, Ondeck NT, Basques BA, Levine BR, Grauer JN. What is the timing of general health adverse events that occur after total joint arthroplasty? Clin Orthop Relat Res 2017;475:2952-9.

19. Chen BP, Dobransky J, Poitras S, Forster A, Beaule PE. Impact of definition and timeframe on capturing surgery-related readmissions after primary joint ar- throplasty. J Arthroplasty 2017;32:3563-7.

20. McCormack RA, Hunter T, Ramos N, Michels R, Hutzler L, Bosco JA. An analysis of causes of readmission after spine surgery. Spine (Phila $\mathrm{Pa} 1976$ ) 2012;37:1260-6.

21. Bernatz JT, Tueting JL, Anderson PA. Thirty-day readmission rates in orthopedics: a systematic review and meta-analysis. PLoS One 2015;10:e0123593.

22. Elsamadicy AA, Ren $\mathrm{X}$, Kemeny $\mathrm{H}$, et al. Independent associations with 30- and 90-day unplanned readmissions after elective lumbar spine surgery: a national trend analysis of 144123 patients. Neurosurgery 2019;84:758-67. 